\title{
Endometriosis of the liver containing mullerian adenosarcoma: Case report
}

\author{
John E. Jelovsek, MD, ${ }^{a}$ Charles Winans, MD, ${ }^{b}$ Jennifer Brainard, MD, ${ }^{c}$ \\ Tommaso Falcone, $M^{a}$
}

\author{
Departments of Obstetrics and Gynecology, ${ }^{\mathrm{a}}$ Surgery, ${ }^{\mathrm{b}}$ and Pathology, ${ }^{\mathrm{c}}$ The Cleveland Clinic Foundation \\ Cleveland, Ohio
}

Received for publication January 28, 2004; revised April 12, 2004; accepted May 19, 2004

\section{KEY WORDS}

Endometriosis

Adenosarcoma

Liver

Postmenopausal

Leuprolide acetate

\begin{abstract}
We present a case of a liver endometrioma in a postmenopausal woman. After failed management with leuprolide acetate, the mass was resected and contained focal areas of mullerian adenosarcoma. This is a rare case of mullerian adenosarcoma that appeared to arise within an endometrioma of the liver.

(c) 2004 Elsevier Inc. All rights reserved.
\end{abstract}

Extrapelvic endometriosis has been reported in the female genitourinary system, large and small bowel, umbilicus, lymph nodes, upper abdomen or upper peritoneum, and liver. Malignancy arising in endometriosis is rare and occurs in less than $1 \%$ of cases. Approximately $75 \%$ of neoplasms complicating endometriosis arise in the ovary. We present a case of endometriosis involving the liver parenchyma with malignant transformation to mullerian adenosarcoma in a patient who had a history of a total hysterectomy and bilateral salpingo-oophrectomy.

\section{Case report}

A 52-year-old woman, gravida 1, para 1, was referred for a liver mass. Eight years ago she underwent a total abdominal hysterectomy with bilateral salpingooophrectomy for menorrhagia at which time she received a diagnosis of endometriosis. Operative reports were not

Reprints not available from the authors. available. Postoperatively, she was placed on an unknown regimen of hormone replacement for hot flashes. After her surgery, she gradually decreased the use of exogenous estrogens during a 5-year period. Three years ago she presented to her primary care provider with flulike symptoms, right-sided shoulder pain, and pain with inspiration. A computed tomographic (CT) scan was performed that showed an $11 \times 7 \mathrm{~cm}$ mass in her liver. Biopsy at the time revealed endometriosis. She was treated with monthly leuprolide acetate injections that shrank the mass to approximately $2 \mathrm{~cm}$ in greatest diameter. No follow-up was received for approximately 3 years until the patient presented to an orthopedic surgeon for chronic shoulder pain. She was prescribed steroid injections and muscle relaxants without relief.

Last year she again had flu-like symptoms. A chest radiograph from her local emergency department was interpreted as pneumonia. Repeat CT scans revealed, "marked progression of a large mixed density posterior perihepatic/capsular mass with apparent direct extension into and invasion of the posterior aspect of the right hepatic lobe." She was assumed to have recurrent 


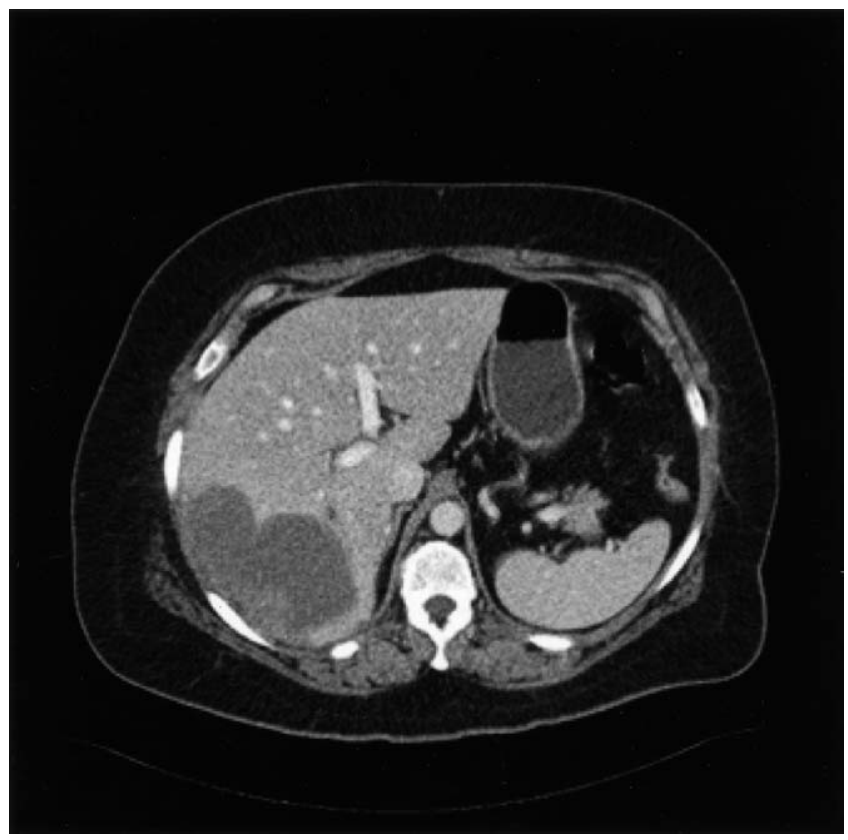

Figure 1 Hepatic mass. The mass involves the right lobe of the liver, diaphragm, and surrounds the upper pole of the right kidney.

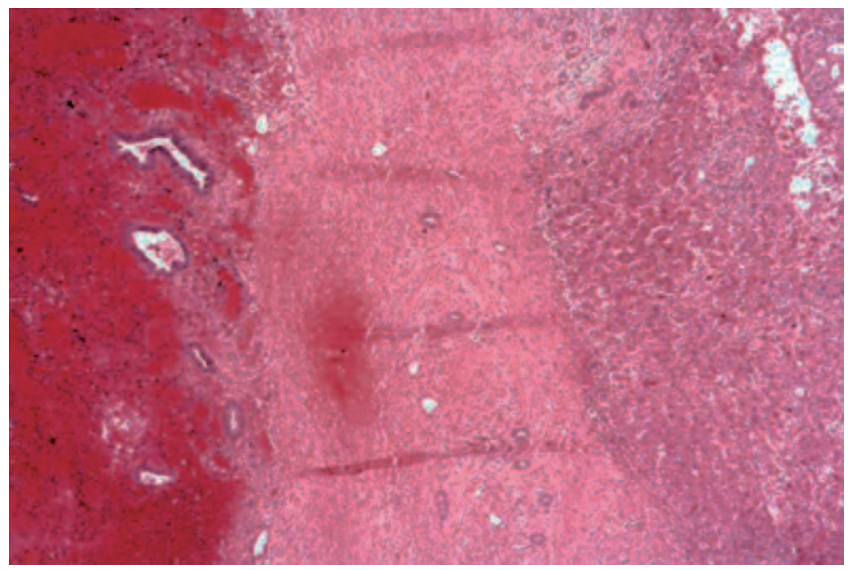

Figure 2 Normal liver parenchyma separated from residual endometriosis by a zone of fibrous tissue.

endometriosis, placed back on leuprolide acetate injections, and referred to our institution for discussion of management options.

At presentation to our clinic, serum follicle-stimulating hormone (FSH) and 17-beta estradiol assays were performed and showed FSH $=5.2 \mathrm{mLU} / \mathrm{mL}$ and estradiol $=22 \mathrm{pg} / \mathrm{mL}$. Repeat ultrasound showed a $2-\mathrm{cm}$ mass in the pelvis, suspicious for ovarian remnant. Repeat CT scan was performed at our institution and interpreted as, "perihepatic, low attenuation mass which by history is consistent with an endometrioma. It has decreased in size when compared with the outside CT" (Figure 1). The mass measured $11.5 \times 7.3 \mathrm{~cm}$. After a 5-

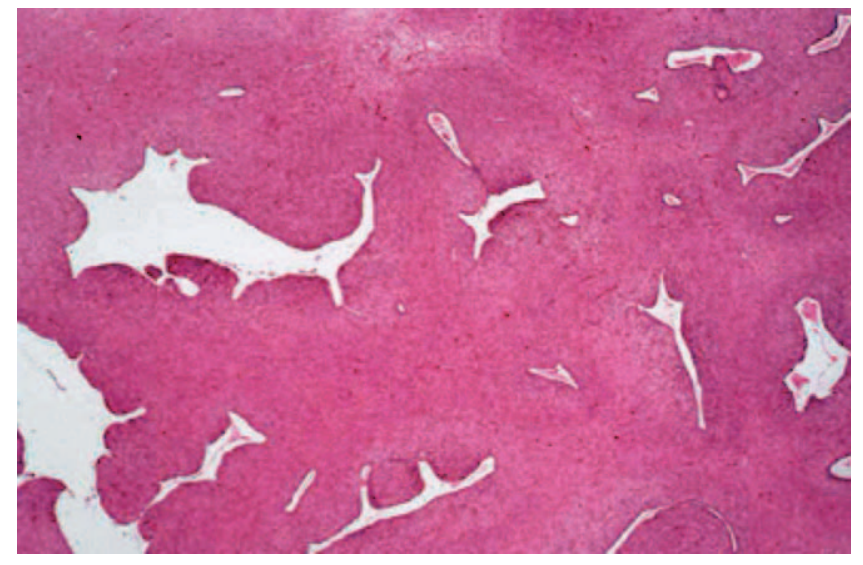

Figure 3 Mullerian adenosarcoma characterized by a leaflike growth pattern, cystically dilated glands lined by benign endometrial-type epithelium surrounded by cellular cuffs of malignant spindle cell stroma. Focally, the stroma has periglandular hypercellularity, mitoses, and low-grade nuclear atypia. These changes are diagnostic of mullerian adenosarcoma.

month treatment with of leuprolide acetate, a repeat CT scan revealed no significant decrease in the size of the mass.

The patient underwent resection of the suspected liver endometrioma, diaphragmatic reconstruction, and pelvic laparotomy with peritoneal biopsies. There was no evidence of ovarian remnant or pelvic endometriosis at laparotomy. Pathologic findings of the liver mass showed cystic and polypoid endometriosis of the liver (Figure 2) and retroperitoneum with contents of the endometrioma from the right lobe of the liver found to harbor focal areas of mullerian adenosarcoma (Figure 3). Pelvic peritoneal biopsy specimens showed fibrosis with no ovarian tissue.

The postoperative management was uncomplicated with only mild elevation of her liver enzymes. Repeat CT scans showed no pelvic mass. Because of the generously negative margins, no adjuvant therapy was recommended. Because there is a lack of standard care for adjuvant therapies, we would reserve high-dose progestins only for recurrence, as it is only cytostatic and not cytotoxic. The risk/benefit profile was not optimal for use in the adjuvant setting.

\section{Comment}

Cases of extrapelvic endometriosis have been reported in the gastrointestinal tract, urinary tract, pulmonary, musculoskeletal, peripheral, and central nervous system, and even in men. However, there have been only 2 reported cases of endometriosis involving the parenchyma of the liver.

Although endometriosis is significantly prevalent in the reproductive female population, it has been esti- 
mated to also be prevalent in $2 \%$ of postmenopausal women. ${ }^{1}$ The FSH and estradiol levels were low in this patient caused by the effect of leuprolide acetate. In our laboratory, estradiol level of $22 \mathrm{pg} / \mathrm{mL}$ is considered castrate levels. Sources of estrogen in the postmenopausal patient arise from the cytochrome p450 enzyme aromatase. Aromatase is primarily expressed in ovarian granulosa cells, placental syncytiotrophoblast, adipose tissue, skin fibroblasts, and the brain. Aromatase messenger RNA has been found in extraovarian endometriotic implants. The aromatase system may be one source of estrogen in cases of postmenopausal endometriosis. The endometriotic implants may be producing enough estrogen to maintain endometrial-like activity.

Hepatic endometriosis containing adenosarcoma has previously been reported. ${ }^{2}$ N'Senda et $\mathrm{al}^{2}$ described a $20 \times 12-\mathrm{cm}$ large mass adhered to the diaphragm. The lower part of the mass was adjacent to the pelvis and the authors assumed that hepatic endometriosis was secondary to primary pelvic endometriosis. In that case, the hepatic endometriosis was incompletely intraparenchymal and appeared to extend from the periphery to the liver. In our patient, an endometrioma was contained in the right lobe of the liver that harbored areas of mullerian adenosarcoma.

Malignant transformation in endometriosis is a rare event with an estimated frequency of $0.3 \%$ to $0.8 \%$. In 1990, Hitti et $\mathrm{al}^{3}$ reviewed the literature regarding malignant transformation of endometriosis. They con- cluded that $90 \%$ of carcinomas were endometrioid and $5 \%$ were clear-cell. Novak and Woodruff ${ }^{4}$ reviewed 35 malignancy cases involving extrapelvic endometriosis. All 35 cases were adenocarcinomas. Endometrial stromal sarcomas as well as malignant-mixed mullerian tumors have been reported in the setting of endometriosis but are exceedingly rare.

In summary, endometriosis should be considered in the differential of a symptomatic liver mass in premenopausal as well as postmenopausal women. Surgical castration was not curative or preventative in this patient. Malignant transformation of endometriosis is an uncommon but possible event and should prompt serious consideration for resection of detected lesions. This case should stimulate new hypotheses regarding the pathogenesis of this disease.

\section{References}

1. Magtibay PM, Heppell J, Leslie KO. Endometriosis-associated invasive adenocarcinoma involving the rectum in a postmenopausal female: report of a case. Dis Colon Rectum 2001;44:1530-3.

2. N'Senda P, Wendum D, Balladur P, Dahan H, Tubiana JM, Arrive L. Adenosarcoma arising in hepatic endometriosis. Eur Radiol 2000;10:1287-9.

3. Hitti IF, Glasberg SS, Lubicz S. Clear cell carcinoma arising in extraovarian endometriosis: report of three cases and review of the literature. Gynecol Oncol 1990;39:314-20.

4. Novak ER, Lambrou CD, Woodruff JD. Ovarian tumors in pregnancy: an ovarian tumor registry review. Obstet Gynecol 1975;46:401-6. 